\title{
JORDAN DERIVATIONS AND ANTIDERIVATIONS OF GENERALIZED MATRIX ALGEBRAS
}

\author{
YANBO Li, LEON VAN WYK AND FENG WEI
}

\begin{abstract}
Let $\mathscr{G}=\left[\begin{array}{cc}A & M \\ N & B\end{array}\right]$ be a generalized matrix algebra defined by the Morita context $\left(A, B,{ }_{A} M_{B},_{B} N_{A}, \Phi_{M N}, \Psi_{N M}\right)$. In this article we mainly study the question of whether there exist the so-called "proper" Jordan derivations for the generalized matrix algebra $\mathscr{G}$. It is shown that if one of the bilinear pairings $\Phi_{M N}$ and $\Psi_{N M}$ is nondegenerate, then every antiderivation of $\mathscr{G}$ is zero. Furthermore, if the bilinear pairings $\Phi_{M N}$ and $\Psi_{N M}$ are both zero, then every Jordan derivation of $\mathscr{G}$ is the sum of a derivation and an antiderivation. Several constructive examples and counterexamples are presented.
\end{abstract}

Mathematics subject classification (2010): 15A78, 16W25, 47L35.

Keywords and phrases: Generalized matrix algebra, Jordan derivation, antiderivation.

\section{REFERENCES}

[1] D. Benkovič, Jordan derivations and antiderivations on triangular matrices, Linear Algebra Appl. 397 (2005), 235-244.

[2] C. BовOC, S. DǍSCǍLESCU AND L. VAN WYK, Isomorphisms between Morita context rings, Linear and Multilinear Algebra, 60 (2012), 545-563.

[3] M. BREŠAR, Jordan derivations on semiprime rings, Proc. Amer. Math. Soc. 104 (1988), 1003-1006.

[4] M. BREŠ AR, Jordan mappings of semiprime rings, J. Algebra 127 (1989), 218-228.

[5] M. BREŠAR, Commuting traces of biadditive mappings, commutativity-preserving mappings and Lie mappings, Trans. Amer. Math. Soc. 335 (1993), 525-546.

[6] M. BReŠAR, Jordan derivations revisited, Math. Proc. Cambridge Philos. Soc. 139 (2005), 411-425.

[7] M. Cohen, D. Fischman And S. Montgomery, Hopf Galosi extensions, smash products, and Morita equivalence, J. Algebra 133 (1990), 351-372.

[8] A. HaGHANY, Hopficity and co-hopficity for morita contexts, Comm. Algebra 27 (1999), 477-492.

[9] D. HAN AND F. WEI, Jordan $(\alpha, \beta)$-derivation on triangular algebras and related mappings, Linear Algebra Appl. 434 (2011), 259-284.

[10] I. N. Herstein, Jordan derivations of prime rings, Proc. Amer. Math. Soc. 8 (1957), 1104-1110.

[11] J.-C. Hou AND X.-F. QI, Generalized Jordan derivation on nest algebras, Linear Algebra Appl. 430 (2009), 1479-1485.

[12] B. E. Johnson, Symmetric amenability and the nonexistence of Lie and Jordan derivations, Math. Proc. Cambridge Philos. Soc. 120 (1996), 455-473.

[13] J.-K. Li And J.-R. Zhou, Characterizations of Jordan derivations and Jordan homomorphisms, Linear Multilinear Algebra 59 (2011), 193-204.

[14] Y.-B. Li AND D. BENKOVIČ, Jordan generalized derivations on triangular algebras, Linear and Multilinear Algebra 59 (2011), 841-849.

[15] Y.-B. LI AND Z.-K. XIAO, Additivity of maps on generalized matrix algebras, Elect. J. Linear Algebra 22 (2011), 743-757.

[16] Y.-B. Li AND F. WEI, Semi-centralizing maps of generalized matrix algebras, Linear Algebra Appl. 436 (2012), 1122-1153.

[17] Y.-B. Li AND F. WEI, Jordan derivations and Lie derivations on path algebras, arXiv: 1203.4925.

[18] F. MA AND G.-X. JI, Generalized Jordan derivations on triangular matrix algebras, Linear Multilinear Algebra 55 (2007), 355-363. 
[19] S. Montgomery, Hopf algebras and their actions on rings, CBMS Regional Conference Series in Mathematics 82, AMS, Providence, R.I., 1993.

[20] K. Morita, Duality for modules and its applications to the theory of rings with minimum condition, Sci. Rep. Tokyo Kyoiku Diagaku Sect. A6 (1958), 83-142.

[21] Z.-K. XIAO AND F. WeI, Jordan higher derivations on triangular algebras, Linear Algebra Appl. 432 (2010), 2615-2622.

[22] Z.-K. XIAO AND F. WeI, Commuting mappings of generalized matrix algebras, Linear Algebra Appl. 433 (2010), 2178-2197.

[23] J.-H. ZhANG, Jordan derivations on nest algebras, Acta Math. Sinica (Chin. Ser.) 41 (1998), 205212.

[24] J.-H. ZHANG AND W.-Y. YU, Jordan derivations of triangular algebras, Linear Algebra Appl. 419 (2006), 251-255. 